\title{
Simulation of microcirculatory hemodynamics: estimation of boundary condition using particle swarm optimization
}

\author{
Qing Pan ${ }^{\mathrm{a}}$, Ruofan Wang ${ }^{\mathrm{b}}$, Bettina Reglin ${ }^{\mathrm{c}}$, Luping Fang ${ }^{\mathrm{a}}$, Axel R. Pries ${ }^{\mathrm{c}, \mathrm{d}}$ and Gangmin \\ Ning, \\ ${ }^{a}$ College of Information Engineering, Zhejiang University of Technology, 310023 Hangzhou, China \\ ${ }^{b}$ Department of Biomedical Engineering, Key Laboratory of Biomedical Engineering of MOE, \\ Zhejiang University, 310027 Hangzhou, China \\ ${ }^{c}$ Department of Physiology and CCR, Charité, Charitéplatz 1, 10117 Berlin, Germany \\ ${ }^{d}$ Deutsches Herzzentrum Berlin, Augustenburger Platz 1, D-13353 Berlin, Germany
}

\begin{abstract}
Estimation of the boundary condition is a critical problem in simulating hemodynamics in microvascular networks. This paper proposed a boundary estimation strategy based on a particle swarm optimization (PSO) algorithm, which aims to minimize the number of vessels with inverted flow direction in comparison to the experimental observation. The algorithm took boundary values as the particle swarm and updated the position of the particles iteratively to approach the optimization target. The method was tested in a real rat mesenteric network. With random initial boundary values, the method achieved a minimized 9 segments with an inverted flow direction in the network with 546 vessels. Compared with reported literature, the current work has the advantage of a better fit with experimental observations and is more suitable for the boundary estimation problem in pulsatile hemodynamic models due to the experiment-based optimization target selection.
\end{abstract}

Keywords: Microcirculation, particle swarm optimization, mathematical model, boundary condition

\section{Introduction}

The hemodynamic characteristics play a central role in regulating microvascular physiological functions, such as tissue perfusion, vascular tone activation, and structural adaptation. Current experimental techniques are limited in investigating the distribution of hemodynamic parameters in microvascular networks because blood flow rates or blood pressures are difficult to measure in every individual vessel. As an alternative approach, mathematical simulation has become prevalent in studying the complex hemodynamics and blood flow regulated mechanotransductive processes for a long time [13]. The mathematical models of microcirculation are usually established based on real microvascular network structures, which are reconstructed from experiments. Intravital microscopy is one of the widely used techniques for reconstructing microvascular networks. Feasible systems are generally flat

\footnotetext{
${ }^{*}$ Corresponding author: Gangmin Ning, Department of Biomedical Engineering, Zhejiang University, Zheda Rd. 38, 310027 Hangzhou, China. Tel.: +86-571-87951091; Fax: +86-571-87951676; E-mail: gmning@zju.edu.cn.
}

0959-2989/14/\$27.50 @ 2014 - IOS Press and the authors. 
tissues, such as mesentery and chicken embryo [4,5]. Recently, with the development of high resolution micro-CT, it is possible to reconstruct more general networks with a three-dimensional structure, such as coronary and tumor tissues [6,7]. These reconstructed networks serve as the base for the theoretical analysis of the blood flow distribution in respective tissues and organs.

In addition to the structural information of the networks, appropriate boundary conditions have to be defined to permit the simulation. The blood flow velocity can be measured in all the boundary vessels by intravital microscopy. However, in our previous simulation, the measured boundary values led to negative blood pressure and inverted flow direction in comparison to the experimental observation [8]. This was probably due to the fact that the vascular system continued to adapt to the environment, resulting in fluctuations in measured boundary values over time. The micro-CT can only acquire the structure of the network rather than the hemodynamic parameters, so that the microcirculatory modeling is hampered by the boundary setting problem.

Efforts have been made to deal with this problem. In our previous study, the boundary values were scaled manually to fit the experimental observation [9]. This approach, while achieving a satisfactory outcome, lacks theoretical support and is therefore difficult to apply to general networks. Fry et al. proposed an optimization method, which aimed to approach the empirical blood pressures and wall shear stresses in all the vessels [10]. The method was superior in modeling large microvascular networks without hemodynamic measurements. However, the effect of optimization was compromised by the exclusion of experimental information. Also, the method was limited in modeling complex hemodynamic conditions, such as pulsatile blood flow in the microcirculation, since the optimization targets were steady state parameters.

In order to improve the performance of boundary estimation and extend its application to pulsatile microcirculatory models, we proposed a novel strategy based on particle swarm optimization (PSO) algorithm. The least number of vessels with inverted flow direction $\left(\mathrm{N}_{\mathrm{INV}}\right)$ in comparison to the experimental observation was taken as the optimization target, because flow direction remains mostly unvaried during experiments. The PSO algorithm was used for deriving the best boundary setting that minimizes the $\mathrm{N}_{\mathrm{INV}}$. The strategy was tested in a rat mesenteric network, in which the optimized results can be compared with the experimental results.

\section{Methods}

\subsection{Network data}

A rat mesenteric network was used in this study as a test platform. Morphological, topological, and hemodynamic data was obtained using intravital microscopy. Details of the experiment were described previously [4]. Briefly, a male Wistar rat was prepared for the intravital microscopy of the mesenteric microcirculation. Fat-free portions of the mesentery were selected for observation with a $\times 25$ salt water immersion objective. The selected area was recorded by video camera for off-line reconstruction. In the preparation procedure, no vascular tone was exhibited. Nevertheless, papaverine $\left(10^{-4} \mathrm{M}\right)$ was continuously superfused to reduce the variation of diameter caused by vascular tone activation. Blood flow velocity was measured for each vessel by computing the frame-to-frame red blood cell moving distance.

The reconstructed network includes 546 segments with 36 boundaries. In this network, blood is primarily perfused by one main arteriole and drained out by one main venule. The other boundaries are indicated as secondary boundaries in this paper. 


\subsection{Hemodynamic model}

The simulation of blood pressure and flow rate in the network was based on the previously developed pulsatile model [9]. In brief, the governing equations were generated for a single vessel following the conservation of mass and momentum as

$$
\left\{\begin{array}{c}
\frac{\partial A}{\partial t}+\frac{\partial(A u)}{\partial x}=0 \\
\frac{\partial u}{\partial t}+u \frac{\partial u}{\partial x}+\frac{1}{\rho} \frac{\partial p}{\partial x}=\frac{2 \pi \mu \alpha}{\rho(1-\alpha)} \frac{u}{A}
\end{array}\right.
$$

where $A$ is the cross-sectional area (CSA), $u$ is the average axial blood flow velocity, $p$ is the average blood pressure over the CSA, $t$ is the time, $x$ is the axial coordinate along the vessel, $\rho$ is the blood density, $\mu$ is the apparent blood flow viscosity, and $\alpha$ is a correction factor that accounts for the velocity profile. Eq. (1) has to be completed by adding a pressure-area relationship

$$
p-p_{0}=\frac{4 \sqrt{\pi} E h}{3 A_{0}}\left(\sqrt{A}-\sqrt{A_{0}}\right)
$$

where $A_{0}$ is the CSA when $p=p_{0}, E$ is the Young's modulus of the vessel wall, and $h$ is the wall thickness. At the junction node, mass conservation and total pressure continuity lead to the relationship

$$
\begin{aligned}
& A_{p} u_{p}=A_{d 1} u_{d 1}+A_{d 2} u_{d 2} \\
& p_{p}+\frac{1}{2} \rho\left(u_{p}\right)^{2}=p_{d 1}+\frac{1}{2} \rho\left(u_{d 1}\right)^{2}=p_{d 2}+\frac{1}{2} \rho\left(u_{d 2}\right)^{2}
\end{aligned}
$$

where the subscripts $p, d 1$ and $d 2$ indicate the parental vessel and its two daughter vessels.

The partial differential equation (PDE) set Eq. (1) was solved using the discontinuous Galerkin (DG) scheme with a spectral/hp spatial discretization. To solve the equation in a one-dimensional domain, the domain was discretized into a mesh of $N$ elements with no overlapping regions. In our study, $N$ was set to one because the diameter was assumed to be uniform along the vessel. In each element, the solution was approximated by a polynomial of degree $P$. The Legendre polynomial was used and $P$ was set to four to ensure the numerical precision. After the discretization and the approximation, the PDE set was converted into an ordinary differential equation (ODE) set. An implicit backward differentiation formula (BDF) solver was adopted to solve the stiff ODE problem. Details of the DG method can be referred in literatures $[11,12]$. 


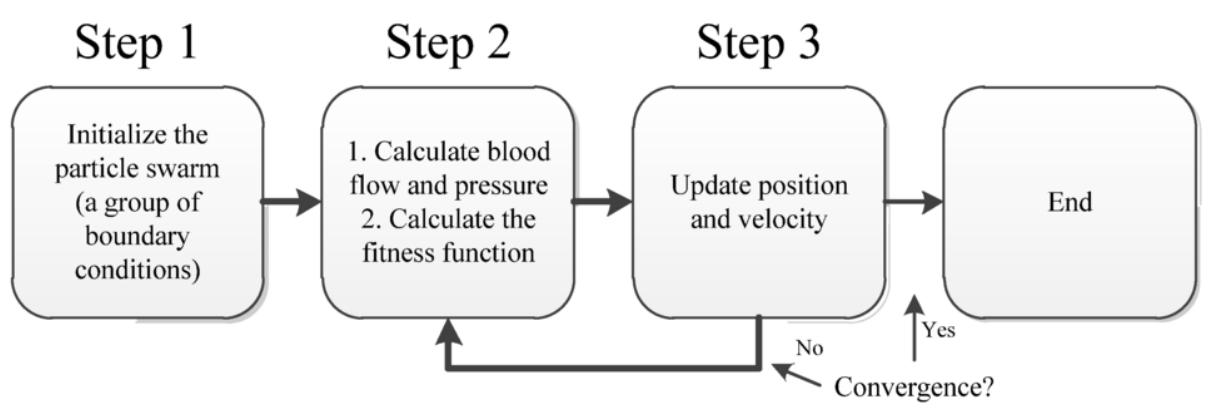

Fig. 1. Flowchart of the PSO optimization procedure.

In the microcirculatory hemodynamic model, several rheological effects have to be taken into account. The blood apparent viscosity is a function of tube diameter and discharge hematocrit due to the non-Newtonian property of the blood in the microcirculation. This is known as the Fahraeus-Lindqvist effect. On the other hand, the phase separation effect, which describes the non-proportional distribution of RBC and plasma at bifurcations, influences the distribution of blood viscosity in the network. The parametric descriptions of the two effects have been introduced in our previous publications [13]. They were applied in the current model.

\subsection{Boundary optimization}

PSO was taken as the optimization strategy in this research. It is an algorithm inspired by the flocking and schooling pattern of birds [14]. It has been widely used in various fields and shows the advantages of fast convergence and high fitness. The optimization procedure is shown in Figure 1. The details of each step are described below.

Step 1: Initialize the particle swarm. The particle swarm is represented as a vector whose size $N_{p}$ is the number of particles in the swarm. The position and velocity of particle $i$ are represented as $P_{i}=\left\{x_{i 1}, x_{i 2}, \ldots, x_{i N_{d}}\right\}$, and $V_{i}=\left\{v_{i 1}, v_{i 2}, \ldots, v_{i N_{d}}\right\}$, where $N_{d}$ is the number of degrees of freedom of the problem. In our case, the vector of blood flow velocity for all the secondary boundaries was selected as the particle. The initial values of the secondary boundaries were randomly given. The main arteriolar input and venular output were excluded, because they determine the level of blood pressure drop of the network. Measured values were prescribed for the two main boundaries.

Step 2: Calculate the fitness function. Fitness function $F\left(P_{i}\right)$ is an objective function that is evaluated for each particle. The optimization algorithm aims to minimize the value of $F\left(P_{i}\right)$. In our problem, the purpose is to achieve the least difference in blood flow direction between the simulation and the experiment. In consequence, $F\left(P_{i}\right)$ was defined as $\mathrm{N}_{\mathrm{INV}}$. Since the flow directions were predetermined according to the experimental observation, $\mathrm{N}_{\mathrm{INV}}$ will increment if the simulated blood flow velocity was negative.

Step 3: Update the position and velocity of the particles. When advancing from one iteration to the next, the position and velocity of the particles are updated following a function that accounts for the deviation between the current and the best particles. The updating function is given as 


$$
\begin{aligned}
& v_{i d}^{k+1}=\omega v_{i d}^{k}+c_{1} \xi\left(p_{i d}^{k}-x_{i d}^{k}\right)+c_{2} \eta\left(p_{g d}^{k}-x_{i d}^{k}\right) \\
& x_{i d}^{k+1}=x_{i d}^{k}+r v_{i d}^{k+1}
\end{aligned}
$$

where $c_{1}$ and $c_{2}$ are global and local accelerations, $\omega$ is the inertia constant, $r$ is the constriction factor and $\xi$ and $\eta$ are uniformly distributed random number ranging between 0 and 1 .

The parameters of the PSO algorithm are listed in Table 1. The optimization procedure was repeated for 20 times.

\section{Results and discussion}

The effect of the optimization was initially examined by checking the $\mathrm{N}_{\mathrm{INV}}$. Figure 2 shows the minimum $\mathrm{N}_{\mathrm{INV}}$ in each iteration. It can be seen that the randomly generated initial swarm results in 213 inverted segments (about $40 \%$ of all the vessels in the network). Optimized by PSO algorithm, $\mathrm{N}_{\text {INV }}$ reduces to 9 from the 8th iteration and remains almost constant thereafter. The optimization result was not influenced by modifying the degree of the polynomial for approximation or by using other ODE solvers, because the flow direction is maintained as long as the numerical convergence was achieved. The proposed method achieved a lower $\mathrm{N}_{\mathrm{INV}}$ in comparison to the research of Fry et al. ( 9 vs. 28). The improvement benefits primarily from the fact that the proposed method took the experimental information $\left(\mathrm{N}_{\mathrm{INV}}\right)$ as the optimization target, while Fry et al. set the optimization target as empirical average blood pressures and wall shear stresses.

Table 1

Parameter setting of the PSO algorithm

\begin{tabular}{llllll}
\hline Parameter & $N_{p}$ & $\omega$ & $c_{1}$ & $c_{2}$ & $r$ \\
\hline Value & 25 & 1 & 4 & 2 & 1 \\
\hline
\end{tabular}

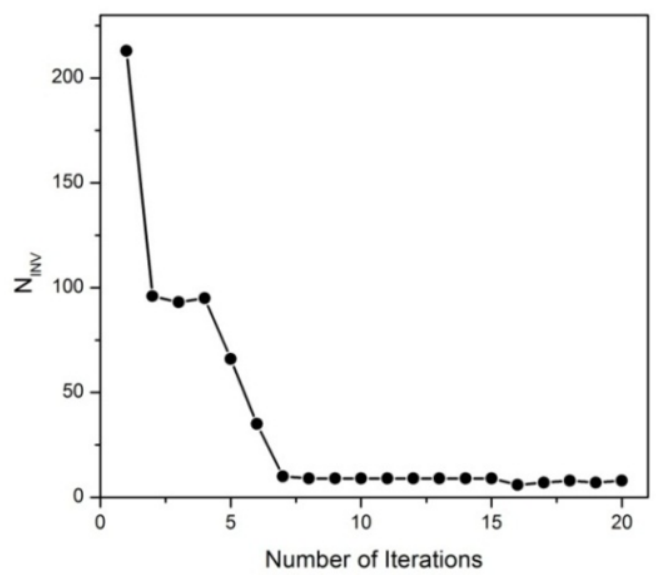

Fig. 2. $\mathrm{N}_{\mathrm{INV}}$ plotted as the function of number of iterations. 
Blood flow rates and blood pressures obtained using the PSO method were compared with the manually optimized boundary conditions results (Figure 3). Essentially, the hemodynamic parameters obtained by the two methods are strongly correlated. Some deviations are observed in the vessels near the secondary boundaries, but these variations arise mostly from the initial boundary setting. In the manual optimization procedure, the boundary values were initiated using measured values, whereas in the PSO method, the initial boundary values were randomly assigned. Although the PSO method fails to approach the measured secondary boundary values, the validity of the model is maintained, because the network is mainly governed by the main feeding arteriole. The effect of the secondary boundaries should be reduced.

Figure 4 shows the distribution of the segments with inverted flow direction. Most of these segments are described by the type of capillary ( 7 out of 9 ). This is reasonable, because capillaries are organized in a meshed rather than tree-like topology. The flow direction in the capillary is sensitive to the structural and topological characteristics of the mesh. In the model, the inverted capillary flow direction might be induced by the inaccurate vessel diameter measurements. Nevertheless, the distribution of hemodynamic parameters will not be significantly modified, because blood perfusion is mainly governed by the feeding arterioles.
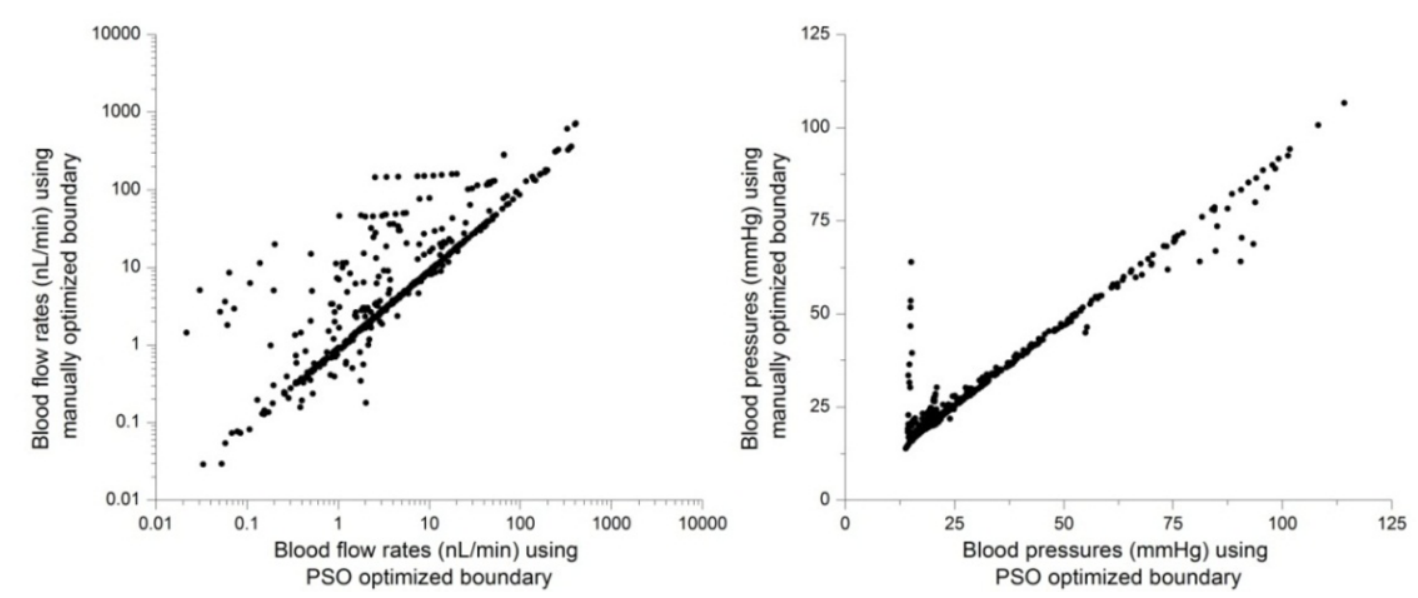

Fig. 3. Comparison of blood flow rates and blood pressures using PSO optimized and manually optimized boundaries.

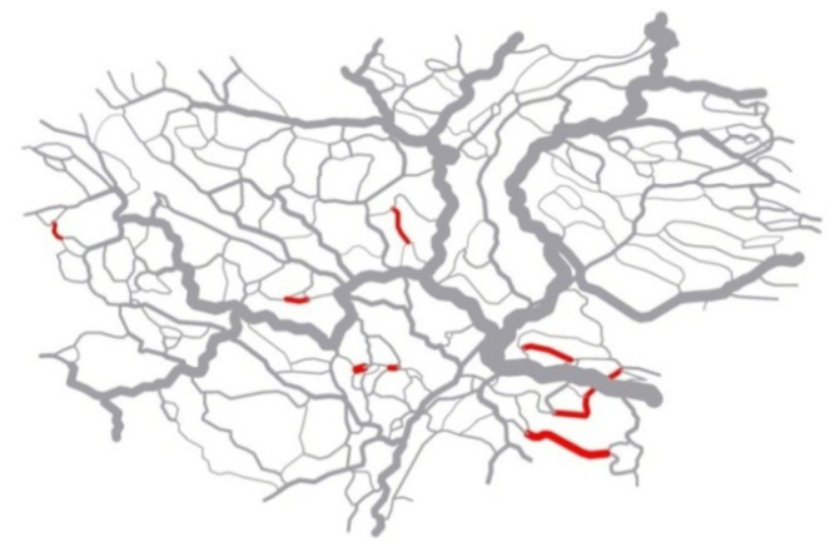

Fig. 4. Map of the microvascular network. The segments showing inverted flow direction are indicated in red. 


\section{Conclusion}

In conclusion, this paper proposed an efficient tool to deal with the boundary setting problem in simulating microvascular hemodynamics. By setting the least $\mathrm{N}_{\mathrm{INV}}$ as the optimization target, the method succeeded in extending the boundary optimization procedure to pulsatile microcirculatory models. Also, the effect of the optimization was improved by involving the experimental information. The improvement will promote the simulation of pulsatile blood flow and pulsatility related physiology in microcirculation.

\section{Acknowledgement}

This research is supported by the National Natural Science Foundation of China (Grant 81271662), Zhejiang Provincial Natural Science Foundation of China (Grant LQ14H180001) and Department of Science and Technology of Zhejiang Province (Grant 2011R50018).

\section{References}

[1] A.R. Pries, M. Höpfner, F. le Noble, et al., The shunt problem: Control of functional shunting in normal and tumour vasculature, Nature Reviews: Cancer 10 (2010), 587-593.

[2] T.W. Secomb, J.P. Alberding, R. Hsu, et al., Angiogenesis: An adaptive dynamic biological patterning problem, PLoS Computational Biology 9 (2013), e1002983.

[3] K. Bartha and H. Rieger, Vascular network remodeling via vessel cooption, regression and growth in tumors, Journal of Theoretical Biology 241 (2006), 903-918.

[4] A.R. Pries, K. Ley and P. Gaehtgens, Generalization of the fahraeus principle for microvessel networks, American Journal of Physiology: Heart and Circulatory Physiology 251 (1986), H1324-1332.

[5] I. Buschmann, A.R. Pries, B. Styp-Rekowska, et al., Pulsatile shear and Gja5 modulate arterial identity and remodeling events during flow-driven arteriogenesis, Development 137 (2010), 2187-2196.

[6] J. Lee, P. Beighley, E. Ritman, et al., Automatic segmentation of 3D micro-CT coronary vascular images, Medical Image Analysis 11 (2007), 630-647.

[7] S.K. Stamatelos, E. Kim, A.P. Pathak, et al., A bioimage informatics based reconstruction of breast tumor microvasculature with computational blood flow predictions, Microvascular Research 91 (2014), 8-21.

[8] A.R. Pries, T.W. Secomb, P. Gaehtgens, et al., Blood flow in microvascular networks, Experiments and Simulation, Circulation Research 67 (1990), 826-834.

[9] Q. Pan, R. Wang, B. Reglin, et al., A one-dimensional mathematical model for studying the pulsatile flow in microvascular networks, Journal of Biomechanical Engineering 136 (2014), 011009:1-11.

[10] B.C. Fry, J. Lee, N.P. Smith, et al., Estimation of blood flow rates in large microvascular networks, Microcirculation 19 (2012), 530-538.

[11] S.J. Sherwin, V. Franke, J. Peiró, et al., One-dimensional modelling of a vascular network in space-time variables, Journal of Engineering Mathematics 47 (2003), 217-250.

[12] G. Karniadakis and S.J. Sherwin, Fundamental concepts in one dimension, in: Spectral/hp Element Methods for Computational Fluid Dynamics, G.H. Golub, ed., Oxford University Press, New York, 2005, pp. 16-80.

[13] A.R. Pries and T.W. Secomb, Microvascular blood viscosity in vivo and the endothelial surface layer, American Journal of Physiology: Heart and Circulatory Physiology 289 (2005), H2657-H2664.

[14] R.C. Eberhart and Y.H. Shi, Particle swarm optimization: Developments, applications and resources, Proceedings of the 2001 Congress on Evolutionary Computation 1 (2001), pp. 81-86. 